\title{
2-Point Error Estimation Algorithm for 3D Thigh and Shank Angles Estimation Using IMU
}

\author{
Yi Chiew Han, Student Member, IEEE, Kiing Ing Wong, Member, IEEE, and Iain Murray, Senior \\ Member, IEEE
}

\begin{abstract}
Inertial Measurement Units (IMUs) have been widely used for many applications such as aircrafts, gaming, animation, and sports. However, the use of IMU in medical diagnosis is still largely unexplored. A great deal of researcher has focused on estimating the pitch angle using accelerometer and gyroscope, while some researchers managed to estimate the roll angle, not many researchers have extended their research to estimate the yaw angle. For those who estimate yaw angle, the utilization of magnetometer is common, but it requires a more complex algorithm to tackle the magnetic interference. This paper presents an innovative method, called the 2-Point Error Estimation Algorithm, to estimate the pitch, roll and yaw angles using accelerometer and gyroscope only. The proposed algorithm is also very computationally efficient to estimate $3 \mathrm{D}$ angles as only two "atan2()" functions are computed throughout the whole walking motion, and 7 additions and 4 multiplications for each angle estimated. The accuracy of the $3 \mathrm{D}$ angles estimated using IMU is validated against gold standard Vicon Optical Motion Capture System. The proposed algorithm gives a low average root mean square error (RMSE) of $2.9^{\circ}, 3.6^{\circ}$, and $4.2^{\circ}$ for flexion/extension (pitch), adduction/abduction (roll), and internal/external rotation (yaw) angles of the thigh and shank, respectively. The proposed algorithm can be used to design a low cost 3D gait evaluation system.
\end{abstract}

Index Terms- Compensation, gyroscope, inertial measurement unit (IMU), gait, Vicon.

\section{INTRODUCTION}

A CCORDING TO THE World Health Organization, about $15 \%$ of the world's population has disability, of which about 3\% suffer significant functional problems such as a walking problem [1]. The assessment of walking can help clinicians to determine and provide optimal care and treatments to patients [2]. To perform gait analysis, the fundamental part is the estimation of joint angular displacement, and it involves the detection of joint position and orientation [3]. Currently, the gait evaluation is done based on visual inspection, but this inspection is subjective and depends on the experience of the medical personnel [4]. Although Vicon Optical Motion System can be used to make an accurate estimation of $3 \mathrm{D}$ orientation

Y. C. Han, and K. I. Wong are with the Department of Electrical and Computer Engineering, Curtin University Malaysia (e-mail: yc.han@postgrad.curtin.edu.my, wong.kiing.ing@curtin.edu.my).

I. Murray is with the School of Electrical Engineering, Computing and Mathematical Sciences, Curtin University Australia (e-mail: I.Murray@curtin.edu.au). angles of human body part [5]. The optical system is non-portable, expensive, and complex to set up due to it requires an empty space without any optical obstruction. Therefore, there is a need to create an Inertial Measurement Unit (IMU) which is portable, affordable and easy-to-setup to estimate the 3D orientation angles.

An IMU is an electronic device that consists of triaxial accelerometers, triaxial gyroscopes, and sometimes magnetometers to estimate 3D angles [6]. The challenge of estimating angles using IMU is that the inertial sensors are prone to drift, noise and surrounding interference such as temperature [7]. Most researchers have performed normalization or filtration of the sensor data to estimate the angles accurately [8], [10]-[14]. Some researchers tilted the accelerometer to read the reference value of each axes at $1 \mathrm{~g}$, i.e. to read when pointing an axis vertically upwards, and offset at $0 \mathrm{~g}$ to use this information to normalize the accelerometer data before starting to estimate orientation angles [8]. The authors tested this method using various models of smartphones and observed that the estimated angles averagely are improved up to $65 \%$. Besides, the authors revealed that the normalization parameters could not be exported from one sensor to another, though of the same model [8].

Tong and Granat [9] used force sensors under the foot to detect mid-stance and use it to remove gyroscope integration drift. When the foot contacted the ground, the algorithm automatically reset the flexion/extension angle estimated using gyroscope to $0^{\circ}$. The estimated flexion/extension angle had a correlation coefficient of 0.97 , and a RMSE of $4.17^{\circ}$. On the other hand, Abhayasinghe and Murray [10] compensated the gyroscope integration drift by updating the angle using accelerometer data when the gyroscope reads small angular velocity and when the accelerometer readings are reliable, i.e. its magnitude is close to $1 \mathrm{~g}$. This results in a high mean correlation of $99.58 \%$ for the flexion/extension angle of the thigh between IMU and Vicon system. However, the authors have yet to estimate the adduction/abduction and internal/external rotation angles of the lower limb.

Luinge and Veltink [11] designed and evaluated Kalman Filter that fuses accelerometer and gyroscope data. The authors observed that the forearm inclination estimate from Kalman Filter is within $3^{\circ}$ RMSE which is significantly smaller than the errors obtained by accelerometer alone which is within $10^{\circ}$ RMSE. However, this technique is not possible to estimate yaw orientation accurately. This is because accelerometer contains 
information about inclination only, and not yaw. Hence, GPS and magnetometer are used to estimate the yaw angle [12].

Madgwick, Harrison \& Vaidyanathan [13] used a method called Gradient Descent based Orientation Filter to estimate the 3D orientation angles of an IMU which consists of an accelerometer, a gyroscope, and a magnetometer. They achieved the RMSE of pitch, roll, and yaw angles as low as $0.625^{\circ}, 0.668^{\circ}$, and $1.110^{\circ}$, respectively. Roetenberg et al. [14] also used an accelerometer, a gyroscope and a magnetometer to estimate 3D angles. The authors took the magnetic disturbance error into account in estimating the orientation angles, and Kalman Filter to minimize the magnetometer data processing in order to compensate the magnetic disturbance. When the magnetic field was disturbed by an iron object, the estimated angle of the method with magnetic disturbance compensation achieved a low mean error of $1.5^{\circ}$, as compared to a big error up to $40^{\circ}$ without using the compensation method.

Although gyroscope can only measure the rate of change of the yaw angle [12], some researchers managed to estimate yaw angles accurately by using gyroscope only. For example, Wu et al. [15] actively changed the orientation of the gyroscope to obtain relations between the gyroscope bias and tilt angle error, so that the gyroscope bias can be estimated and compensated. Using this method, the yaw angle drift, sometimes also known as the heading angle drift, was less than $4^{\circ}$ after an hour, while the heading angle drift without using this method was more than $15^{\circ}$ after an hour. Besides, Bonnet et al. [16] used a single gyroscope and a combination of Fourier Linear Combiner (FLC) and weighted FLC to estimate the 3D orientation angles of trunk. The authors compared the phase of measured angular velocity from gyroscope with the estimated angular velocity, and then modified the frequency weight to reduce the angle estimation error. The estimated 3D angles had RMSE less than $1.0^{\circ}$, and correlation coefficient of about 0.85 .

Some researchers [14] [17] tested their algorithms by rotating the IMU gradually, and achieved low RMSE and high correlation coefficient. This shows that the algorithms are suitable to estimate the inclination of a static or slow-moving object. It is likely to have higher error when the IMU is used for estimating walking motion because there is a significant acceleration due to motion, especially during heel strike and toe-off [18].

Other than being used to estimate the orientation angles, it is worthwhile to note that the IMUs can also be used for indoor positioning. Do et al. [19] utilized vertical acceleration to estimate the horizontal displacement and achieved low average error of $1 \%$ for straight line paths. Yoon et al. [20] fused data from ultra-wideband system and IMUs to track the spatial location and motion of a human.

This paper presents an innovative method to estimate the 3D orientation angles of the thigh and shank using IMUs. The proposed algorithm estimates the errors of the gyroscope angles at the starting and stopping time of a walking motion, and linearly distribute these two errors to compensate the gyroscope drift. The key merit of the proposed algorithm is that it is more computationally efficient than most other methods [10]-[16], while still achieving low average RMSE of $3.6^{\circ}$ for pitch, roll, and yaw angles.

The remainder of this paper is structured as follows. Data collection method is discussed in Section II. The 3D angles estimation algorithm using IMU is described in Section III. Experimental results and discussion are presented in Section IV.

\section{DATA COLLECTION}

\section{A. Inertial Measurement Unit (IMU)}

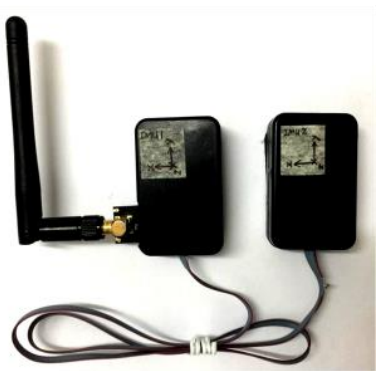

(a)

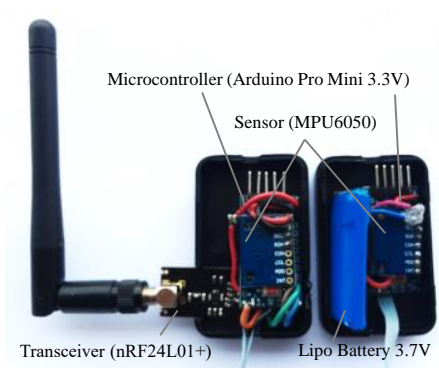

(b)
Fig. 1. IMUs. (a) External view. (b) Internal view.

Two low-cost wireless IMUs, as shown in Fig. 1, were constructed. Each IMU has a sensor (MPU6150) which consists of a tri-axis accelerometer with $\pm 2 \mathrm{~g}$ range and a tri-axis gyroscope with $\pm 250^{\circ}$ /s range, both in 16-bit resolution. The sensors were sampled at $100 \mathrm{~Hz}$. The IMU also consists of a microcontroller Arduino Pro Mini 3.3V, a wireless transceiver nRF24L01+, and a 3.7V Lipo battery. At the dongle side, another Arduino Pro Mini 3.3V received data from the IMU wirelessly using the transceiver nRF24L01+ and the data was transferred to a computer instantly.

All electronic components used in this research could easily be purchased from eBay. The total cost of the electronic components including two IMUs and a dongle was about USD15.

\section{B. Experimental Setup}
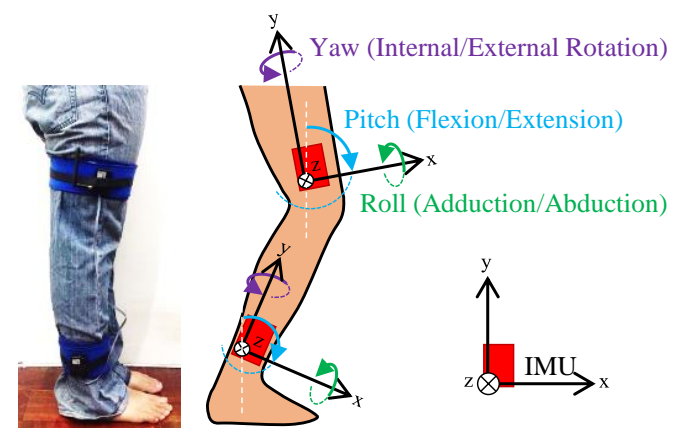

Fig. 2. Flexion/extension, adduction/abduction, and internal/external rotation of thigh and shank.

The flexion/extension, adduction/abduction and internal/external rotation angles of the thigh and shank, as shown in Fig. 2, are also commonly known as the pitch, roll and yaw angles, respectively. The primary motion of the leg is 
flexion/extension, while adduction/abduction and internal/external rotation also occur but the amplitude are less consistent among healthy individual due to soft tissue and bony constraints [21]. However, this may not be the case for people with leg problem, therefore it is needed to estimate the 3D angles of the thigh and shank, instead of just the flexion/extension angle.

The 3D angles estimated using IMUs were validated against the gold standard Vicon Optical Motion Capture System with 18 cameras at sampling rate of $300 \mathrm{~Hz}$. Fig. 2 shows that the IMUs were strapped at the outer side of the thigh and shank using Velcro straps without any skin penetration. The markers of the Vicon system are placed on the IMUs. The 3D movement of the thigh and shank were tracked simultaneously using IMU and Vicon system.

The subjects were requested to stand still for 3-5 seconds, then walk straight on a flat surface for 3 to 5 steps, and finally stood still for another 3-5 seconds. Although more steps were always preferred per trial, 3 to 5 walking steps would be sufficient to capture the walking pattern of an individual for gait analysis as walking is a repetitive motion. Besides, if the application of this research is for gait rehabilitation, a patient may just be able to walk for 3 to 5 steps. 60 walking trials were collected from 10 healthy adults. For the first 3 trials, each volunteer walked straight normally. For another 3 trials, the volunteers were requested to walk straight but intentionally moving their legs outwards in each walking cycle to simulate abnormal walking.

\section{DATA PROCESSING}

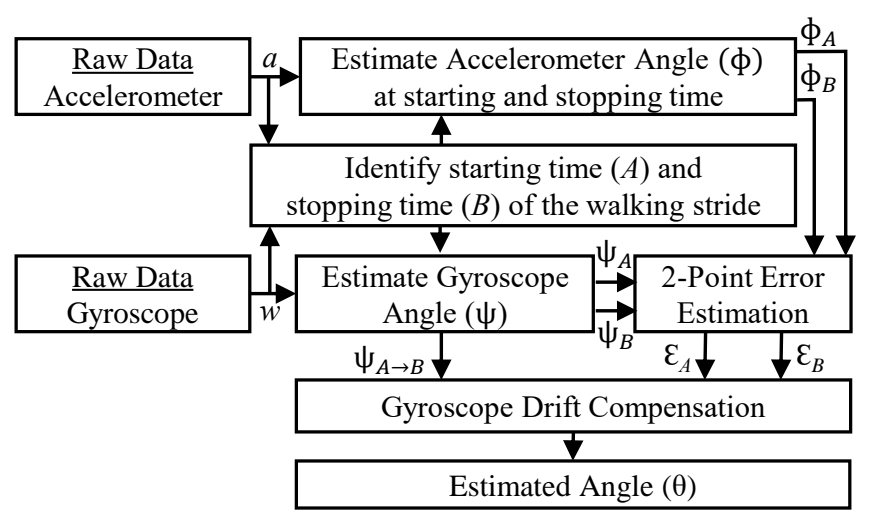

Fig. 3. Flowchart to estimate Flexion/Extension and Adduction/Abduction angles.

\begin{tabular}{|c|c|c|}
\hline Raw Data \\
Accelerometer
\end{tabular}

Fig. 4. Flowchart to estimate Internal/External Rotation angle.

\section{A. Identify the Starting and Stopping Time of Walking}

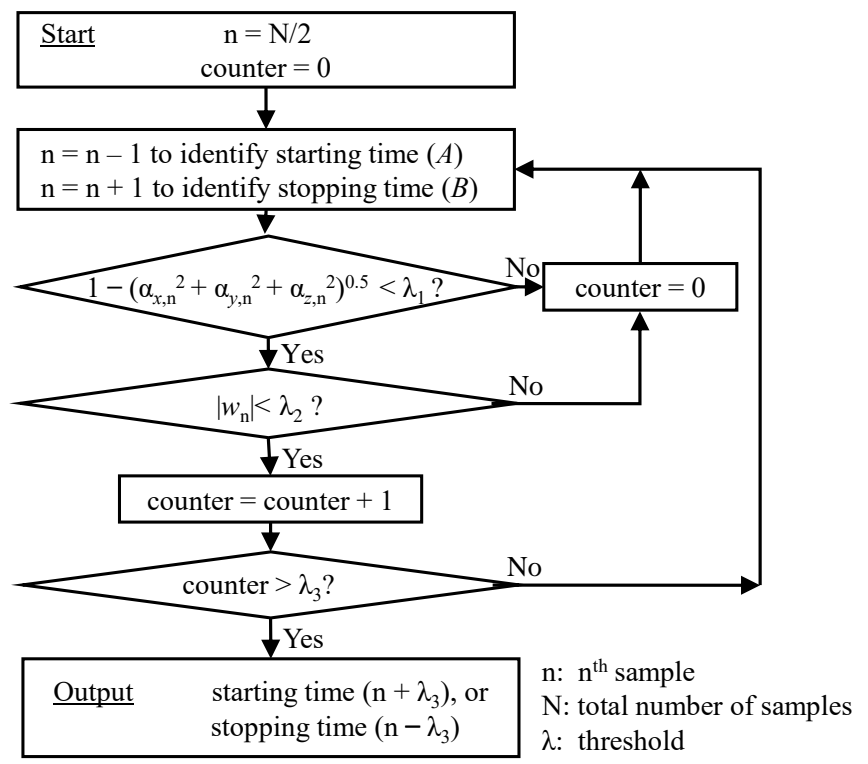

Fig. 5. Flowchart to identify the starting or stopping time of walking.

The 2-Point Error Estimation algorithm developed requires the starting and stopping time of walking to be known. The starting and stopping time can be recorded manually using a timer before and after each walking movement. However, manual time record is tedious and prone to human error of recording the time earlier or later than the actual time. For a more accurate identification of the starting and stopping times of waking movement, it is necessary to create an algorithm to do so automatically.

To utilise accelerometer and gyroscope to identify the starting and stopping time of a walking movement, it is necessary to know the parameters that are measured by the two inertial sensors. The measured accelerometer data $a$ can be modelled as (1) where $g$ denotes the gravitational acceleration, $M$ denotes the linear acceleration due to movement, $\mu_{a}$ denotes the accelerometer DC offset plus noise. The measured gyroscope data $w$ is modelled as (2) where $\Omega$ denotes the angular velocity, $\mu_{w}$ denotes the gyroscope DC offset plus noise.

$$
\begin{gathered}
a=g+M+\mu_{a} \\
w=\Omega+\mu_{w}
\end{gathered}
$$

When the person is standing still, the linear motion $M$ is minimized, and hence accelerometer will measure $a \approx g+\mu_{a}$. On the other hand, when the person is standing still, gyroscope will ideally measure zero angular velocity $\Omega$, and hence $w \approx \mu_{w}$. Assuming the DC offset and noise of the accelerometer and gyroscope noise are negligible, the measured values are now left with $a \rightarrow g$, and $w \rightarrow 0$.

The Earth's gravitational acceleration is $1 \mathrm{~g}$ and pointing vertically downwards, so only the vertical axis of the accelerometer would only measure $1 \mathrm{~g}$ at vertical axis, while the 
other two horizontal axes would measure $0 \mathrm{~g}$, i.e. $|a|=\left(a_{\mathrm{x}}{ }^{2}+a_{\mathrm{y}}{ }^{2}\right.$ $\left.+a_{\mathrm{z}}^{2}\right)^{0.5} \rightarrow 1 \mathrm{~g}$, where $x, y$, and $z$ are the axes of the sensor. For the gyroscope, all three axes would ideally measure $0 \%$ s. By fulfilling eqs. (3-4), the period of which the person is standing still can be identified, where $\lambda_{1} \rightarrow 0$ and $\lambda_{2} \rightarrow 0$. The recommended value for $\lambda_{1}$ is $0.07 \mathrm{~g}$, while $\lambda_{2}$ is recommended to be $10 \%$ s due to the offset and noise of the sensors.

$$
\begin{gathered}
1-\left|\sqrt{a_{x}^{2}+a_{y}^{2}+a_{z}^{2}}\right|<\lambda_{1} \\
\left|w_{i}\right|<\lambda_{2} \text { for } i=x, y, z
\end{gathered}
$$

Fig. 5 shows the flowchart to identify the starting and stopping time of walking. The algorithm checks eqs. (3-4) from the middle of the dataset which is when the person is walking, and progress towards the start or end of walking. When eqs. (3-4) are first fulfilled for a continuous number of $\lambda_{3}$ time, the starting or stopping time can be identified. The threshold $\lambda_{3}$ is set to be 50 in this research.

It must be noted that the ideas of eqs. (3-4) are taken from [10], but the researchers used these equations to decide whether to use accelerometer or gyroscope data to estimate the flexion/extension angle, while the application of these two equations in this proposed method is to identify the starting and stopping time of walking.

\section{Estimation of Accelerometer and Gyroscope Angles}

Accelerometer can estimate the pitch angle $\phi_{\text {pitch }}$ and roll angle $\phi_{\text {roll }}$ according to (5) and (6), respectively. However, accelerometer cannot estimate the yaw angle $\phi_{\text {yaw }}$ because accelerometer measures only the gravitational acceleration which is a vertical reference, but the estimation of the yaw angle requires a horizontal reference.

$$
\begin{gathered}
\phi_{\text {pitch }}=\operatorname{atan} 2\left(a_{x}, a_{z}\right) \\
\phi_{\text {roll }}=\operatorname{atan} 2\left(a_{y}, a_{z}\right)
\end{gathered}
$$

In this research, the accelerometer data is low pass filtered using moving average in (7). The recommended value of $K$ is 30 .

$$
\bar{a}_{n}=\frac{1}{2 K} \sum_{i=n-K}^{n+K} a_{i} \text { for } \begin{gathered}
n<A \\
n>B
\end{gathered}
$$

Gyroscope can estimate the pitch angle $\psi_{\text {pitch }}$, roll angle $\psi_{\text {roll }}$, and yaw angle $\psi_{\text {yaw }}$ using (8) and (9), respectively. The initial gyroscope pitch and roll angles are set as the accelerometer angles as shown in (8), while the initial yaw angle is set as $0^{\circ}$ because the person is facing straight initially. The gyroscope angle can then be estimated using eq. (9) which is the integration of measured angular velocity $w$, where $T$ is the sampling time.

$$
\begin{array}{cc}
\psi_{n}=\phi_{n} & \text { for } n=1 \\
\psi_{n}=\psi_{n-1}+w_{i, n} T & \text { for } \begin{array}{c}
n=2,3,4, \cdots, N \\
i=x, y, z
\end{array}
\end{array}
$$

D. 2-Point Error Estimation and Gyroscope Drift

\section{Compensation}

To compensate the gyroscope drift, a 2-Point Error Estimation algorithm is developed. The error of the estimated gyroscope angle is first calculated at starting time $A$ and stopping time $B$ of walking. The error at these two points are called the 2-point error. To estimate the 2-point error, the gyroscope angle is compared with the accelerometer angle using (10) by calculating their difference $\varepsilon$. The initial (at starting time $A$ ) and final (at stopping time $B$ ) pitch and roll angles of the thigh and shank are measured using (5) and (6).

$$
\varepsilon_{i, n}=\psi_{i, n}-\phi_{i, n} \text { for } \begin{gathered}
n=A, B \\
i=\text { pitch, roll }
\end{gathered}
$$

As accelerometer cannot estimate the yaw angle, the initial yaw angle at time $A$ is assumed to be $0^{\circ}$ because the person is facing straight initially. Since the heading before and after a person walks straight should ideally remain the same, the final yaw angle at time $B$ is also assumed to be $0^{\circ}$. The 2-point error of the gyroscope yaw angle can then be estimated using (11) by subtracting the estimated gyroscope yaw angle with $0^{\circ}$.

$$
\varepsilon_{\text {yaw }, n}=\psi_{\text {yaw }, n} \text { for } n=A, B
$$

The 2-point error calculated in (10) and (11) are then used to estimate the error of the gyroscope angle during walking motion, i.e. from time $A$ to $B$. The error of the gyroscope angle from time $A$ to $B$ is estimated by linearly distributing the 2-point error as in (12) using a linearly distributed weight $W$ calculated in (13). The final estimated 3D angles $\theta$ can be calculated using (14) by subtracting the estimated error from the estimated gyroscope angle.

$$
\begin{gathered}
\varepsilon_{i, n}=\varepsilon_{i, A}\left(1-W_{n}\right)+\varepsilon_{i, B} W_{n} \\
W_{n}=\frac{n-A}{B-A} \\
\theta_{i, n}=\psi_{i, n}-\varepsilon_{i, n} \\
\text { for } \begin{array}{c}
n=A, A+1, A+2, \cdots, B \\
i=\text { pitch, roll , yaw }
\end{array}
\end{gathered}
$$

The pitch and roll angles when the person is standing still, i.e. before starting time $A$ and after stopping time $B$, can be estimated using (5) and (6), respectively. 


\section{RESULTS AND DISCUSSION}
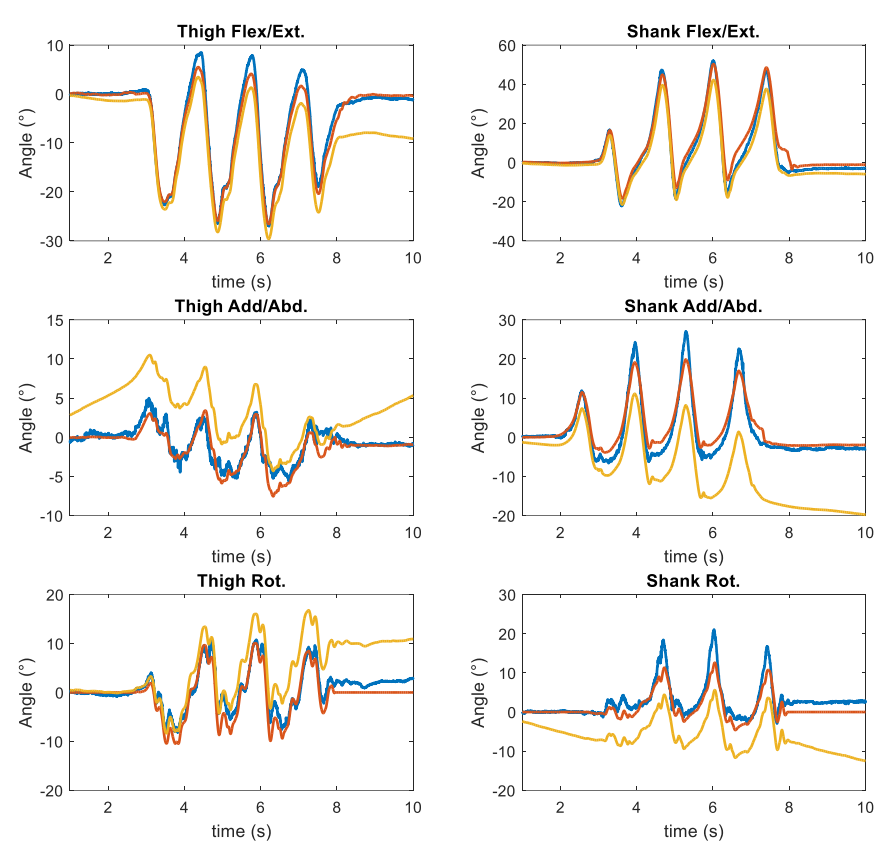

-Vicon Proposed Method

__ Gyroscope angle (eq. 9)

Fig. 6. Estimated 3D angles of the thigh and shank during normal walking on a flat surface.

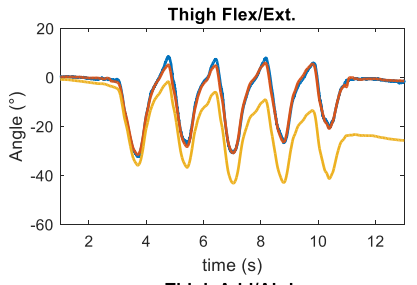

Thigh Add/Abd.

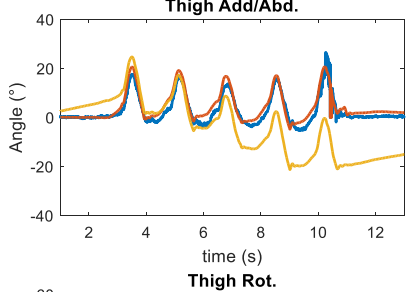

Thigh Rot.

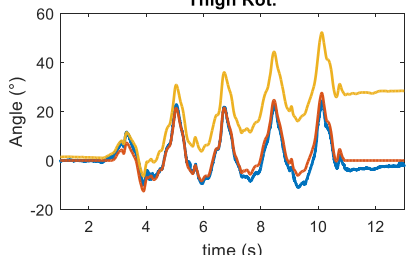

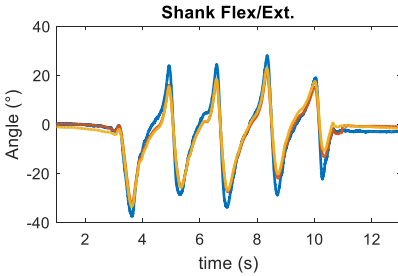

Shank Add/Abd.

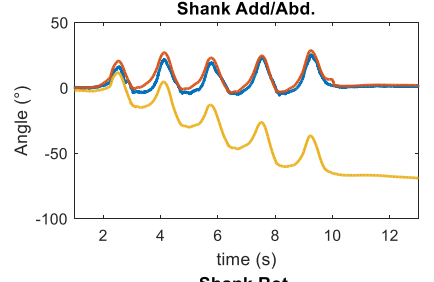

Shank Rot.

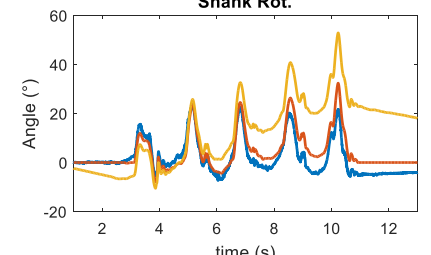

— Vicon $\quad$ Proposed Method —- Gyroscope angle (eq. 9)

Fig. 7. Estimated 3D angles of the thigh and shank during abnormal walking on a flat surface.

Fig. 6 and 7 show the 3D orientation angles of the thigh and shank of the same individual. The blue lines are the angle waveforms estimated using gold standard Vicon Optical Motion Capture System. The red lines are the angle waveforms estimated using the proposed 2-Point Error Estimation algorithm. The yellow lines are the angle waveforms estimated using (9) which is prone to gyroscope integration drift.
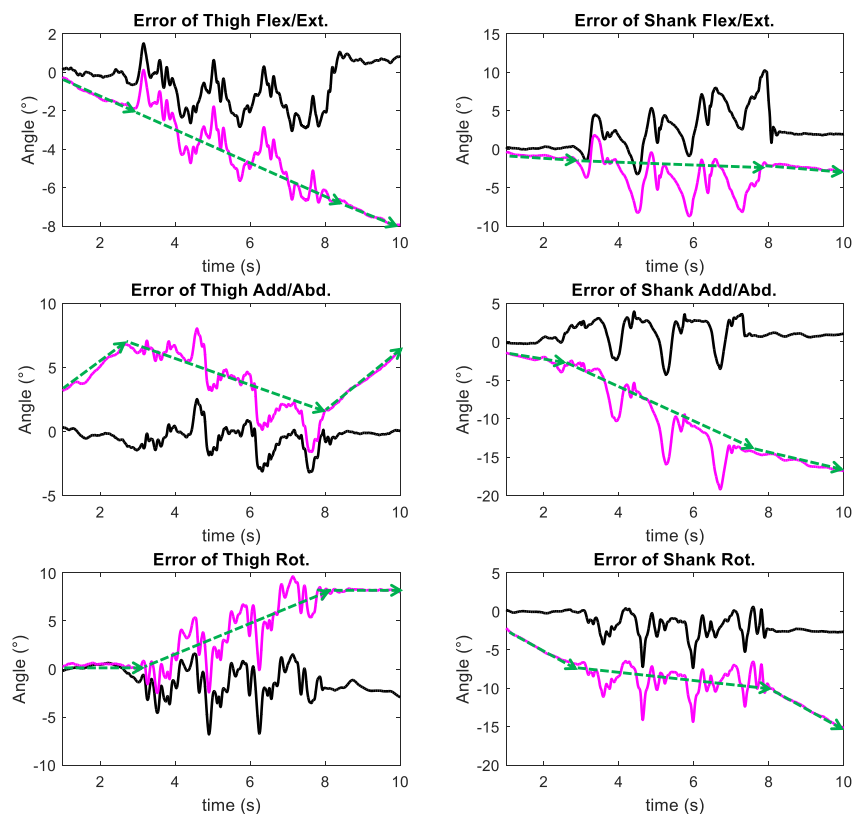

- Proposed Method $\quad$ Gyroscope angle error

--> Estimated Gyroscope Drifting

Fig. 8. Error of the estimated thigh and shank angles during normal walking on a flat surface.

Fig. 8 shows the error of the estimated 3D angles in Fig. 7. The error of the gyroscope angles before and after walking were mostly linear. However, the error of the gyroscope angles (pink lines) was fluctuating while walking. Drawing a straight line (dotted green line) connecting the gyroscope angles at starting time $A$ and stopping time $B$, it was estimated in this research that the drifting direction of the gyroscope angles was almost linear.

Referring to the thigh flex/ext. in Fig. 8, the gyroscope drifting direction was almost linear at all time. However, there are cases, for example the thigh add/abd. in the same figure, that the estimated drifting direction of the gyroscope angles were different before, during, and after walking. Therefore, we proposed a 2-point gyroscope error estimation, i.e. estimating the error based on pre and post-calibration method, while many researchers estimate the sensor error based on pre-calibration method [8].

TABLE I

AVERAGE RoOt MEAN SQUARE ERROR (RMSE) AND CORRELATION COEFFICIENT (CORR) OF THIGH AND SHANK ANGLES ESTIMATED USING PROPOSED 2-POINT ERROR ESTIMATION ALGORITHM

\begin{tabular}{|c|l|c|c|c|c|c|c|}
\hline \hline \multirow{4}{*}{$\begin{array}{c}\text { Walking } \\
\text { on Flat } \\
\text { Surface }\end{array}$} & \multicolumn{2}{|c|}{$\begin{array}{c}\text { Flexion/ } \\
\text { Extension }\end{array}$} & \multicolumn{2}{|c|}{$\begin{array}{c}\text { Adduction/ } \\
\text { Abduction }\end{array}$} & \multicolumn{2}{|c|}{$\begin{array}{l}\text { Internal/ } \\
\text { External } \\
\text { Rotation }\end{array}$} \\
\cline { 3 - 9 } & & Thigh & Shank & Thigh & Shank & Thigh & Shank \\
\hline \multirow{2}{*}{$\begin{array}{c}\text { RMSE } \\
\left(^{(}\right)\end{array}$} & Normal & 1.934 & 3.685 & 2.017 & 4.701 & 3.604 & 4.039 \\
\cline { 2 - 9 } & Abnormal & 2.270 & 3.589 & 2.729 & 4.952 & 4.184 & 4.920 \\
\cline { 2 - 9 } & Overall & 2.102 & 3.637 & 2.373 & 4.826 & 3.894 & 4.479 \\
\hline CORR & Normal & 0.989 & 0.967 & 0.924 & 0.795 & 0.816 & 0.872 \\
\cline { 2 - 8 } & Abnormal & 0.986 & 0.986 & 0.932 & 0.854 & 0.907 & 0.901 \\
\cline { 2 - 8 } & Overall & 0.988 & 0.977 & 0.928 & 0.825 & 0.862 & 0.886 \\
\hline \hline
\end{tabular}


TABLE II

Average RoOT MEAn SQUARE ERROR $\left(^{\circ}\right)$ AND CORRElation COEFFICIENT (CORR) OF THIGH AND SHANK ANGLES ESTIMATED BY INTEGRATING GYROSCOPE DATA AS IN EQ. (8)

\begin{tabular}{|c|l|c|c|c|c|c|c|}
\hline \hline \multirow{4}{*}{$\begin{array}{c}\text { Walking } \\
\text { on Flat } \\
\text { Surface }\end{array}$} & \multicolumn{2}{|c|}{$\begin{array}{c}\text { Flexion/ } \\
\text { Extension }\end{array}$} & \multicolumn{2}{|c|}{$\begin{array}{c}\text { Adduction/ } \\
\text { Abduction }\end{array}$} & \multicolumn{2}{|c|}{$\begin{array}{c}\text { Internal/ } \\
\text { External } \\
\text { Rotation }\end{array}$} \\
\cline { 3 - 8 } & Thigh & Shank & Thigh & Shank & Thigh & Shank \\
\hline \multirow{3}{*}{$\begin{array}{c}\text { RMSE } \\
\left(^{\circ}\right)\end{array}$} & Normal & 7.93 & 6.69 & 11.12 & 23.43 & 7.88 & 14.45 \\
\cline { 2 - 9 } & Abnormal & 14.46 & 10.55 & 13.02 & 36.09 & 16.13 & 18.53 \\
\cline { 2 - 9 } & Overall & 11.20 & 8.62 & 12.07 & 29.76 & 12.00 & 16.49 \\
\hline CORR & Normal & 0.942 & 0.979 & 0.812 & 0.452 & 0.728 & 0.821 \\
\cline { 2 - 8 } & Abnormal & 0.877 & 0.949 & 0.719 & 0.404 & 0.635 & 0.629 \\
\cline { 2 - 8 } & Overall & 0.909 & 0.964 & 0.765 & 0.428 & 0.682 & 0.725 \\
\hline \hline
\end{tabular}

The root mean square error (RMSE) and correlation coefficient in Tables I and II were calculated for the walking period, i.e. from starting time $A$ to stopping time $B$. This is because we are interested to capture the walking pattern, instead of the standing pattern. The results improved when the standing still period was considered.

Referring to Table I, the proposed 2-point error estimation algorithm resulted an averagely low RMSE of less than $5^{\circ}$ for all 3D angles of the thigh and shank, in normal and abnormal walking conditions. Comparing with the angles estimated by directly integrating the gyroscope data, the proposed 2-point error estimation algorithm is proven effective in compensating the gyroscope drift. The RMSE of the angles estimated by directly integrating gyroscope data is $7-36^{\circ}$ as shown in Table II.

The flexion/extension angle estimated using the proposed algorithm achieves high correlation coefficient of $98.8 \%$ for thigh, and $97.7 \%$ for shank. The adduction/abduction and internal/external rotation angles estimated using proposed algorithm achieve lower correlation coefficient of $83-93 \%$ as compared to the flexion/extension angle. However, comparing Tables I and II, the proposed algorithm improves the adduction/abduction and internal/external rotation angles from $43-77 \%$ to $83-93 \%$, i.e. an improvement of $16-40 \%$.

Besides, it has to be noted that filtration of raw gyroscope data is not required in the proposed algorithm. This reduces smoothening or alteration of the angle waveform pattern. The waveform pattern of the estimated 3D angles is relatively similar to that of Vicon system, as shown in Fig. 6 and 7.

This proposed algorithm is also computationally efficient as only two "atan2()" functions are computed in (5) and (6) throughout the whole walking motion, and 7 additions and 4 multiplications for each angle estimated using (9) - (14).

A disadvantage of the proposed algorithm is that it is only suitable to estimate the 3D angles for a short duration of time. However, as walking cycle is expected to be repetitive with slight differences [22], capturing data of 3 to 5 walking steps are sufficient to capture the gait pattern of a person for gait evaluation. Based on the experimental results, the proposed algorithm can at least estimate the $3 \mathrm{D}$ angles accurately up to 15 seconds, which is sufficient to capture 5 walking steps of a person for gait analysis.

Moreover, this proposed method is unable to estimate angles in real time, but real-time angle estimation is not necessary for applications such as gait evaluation.
Furthermore, many researchers utilize magnetometer to estimate yaw angle [13] [14] [23]. The proposed 2-point error estimation algorithm, however, does not require a magnetometer although a magnetometer can be added to improve the accuracy of internal/external rotation angle. As magnetometer is prone to magnetic interference [24], avoiding it has drastically improved the computational efficiency. However, the assumption that the yaw angle remains before and after walking is only true for walking in a straight line. This assumption has resulted a considerably accurate estimation of the yaw angle with low average RMSE of $3.9^{\circ}$ for thigh and $4.5^{\circ}$ for shank.

The proposed algorithm requires the users to stand still before and after walking. As this system is designed for clinical application, it is possible that some patients with impaired gaits would have involuntarily shaky legs [25]. This creates a problem that there is no standing still period for the algorithm to estimate the 2-point error. To solve this, the thresholds in (3) and (4) can be increased to values slightly higher than the average values of accelerometer and gyroscope data collected in the first 1 second, respectively. The increased thresholds would treat the shaky legs as standing still, and then the walking motion could be identified when the thigh flexes/extends above these thresholds.

\section{CONCLUSION}

A method to estimate the 3D angles of the thigh and shank has been proposed. The proposed algorithm is not only accurate, but also computationally efficient and easy to be implemented. In future work, we plan to diagnose normal and abnormal gaits using the estimated 3D angles. It is also worthwhile to investigate the accuracy of the algorithm at different duration so that it could be used for other applications.

\section{ETHICAL APPROVAL}

Ethical approval for this research is granted by Curtin University ethical review committee with approval number HRE2017-0834.

\section{ACKNOWLEDGMENT}

The authors would like to thank Curtin University Bentley Campus for the permission to use Vicon Optical Motion Capture System for this research.

\section{REFERENCES}

[1] E. E. Reber, R. L. Michell, and C. J. Carter, "World Report on Disability," World Health Organization, and World Bank Group, 2011. Available: http://www.who.int/disabilities/world_report/2011/report/en/

[2] Hashimoto, K., Higuchi, K., Nakayama, Y., \& Abo, M. (2007). Ability for basic movement as an early predictor of functioning related to activities of daily living in stroke patients. Neurorehabilitation and Neural Repair, 21(4), 353-357.

[3] A. Muro-de-la-Herran, B. Garcia-Zapirain and A. Mendez-Zorrilla. Gait analysis methods: An overview of wearable and non-wearable systems, 
highlighting clinical applications. Sensors 14(2), pp. 3362-3394. 2014. DOI: http://dx.doi.org.dbgw.lis.curtin.edu.au/10.3390/s140203362.

[4] T. Krosshaug et al, "Estimating 3D joint kinematics from video sequences of running and cutting maneuvers--assessing the accuracy of simple visual inspection," Gait Posture, vol. 26, (3), pp. 378-385, 2007.

[5] D. Knudson, "Fundamentals of Biomechanics". Springer Science \& Business Media, 2013, pp. 10

[6] W. Geiger et al., "MEMS IMU for AHRS applications," 2008 IEEE/ION Position, Location and Navigation Symposium, Monterey, CA, 2008, pp. 225-231. doi: 10.1109/PLANS.2008.4569973

[7] R. E. Mayagoitia, A. V. Nene and P. H. Veltink, "Accelerometer and rate gyroscope measurement of kinematics: an inexpensive alternative to optical motion analysis systems," J. Biomech., vol. 35, (4), pp. 537-542, 2002.

Available: http://www.sciencedirect.com/science/article/pii/S0021929001002317S.

[8] M. De Marsico, D. De Pasquale and A. Mecca, "Embedded Accelerometer Signal Normalization for Cross-Device Gait Recognition," 2016 International Conference of the Biometrics Special Interest Group (BIOSIG), Darmstadt, 2016, pp. 1-5. doi: $\quad$ 10.1109/BIOSIG.2016.7736920 URL: http://ieeexplore.ieee.org/stamp/stamp.jsp?tp=\&arnumber=773692 0\&isnumber $=7736893$

[9] K. Tong and M. H. Granat, "A practical gait analysis system using gyroscopes,"Med. Eng. Phys., vol. 21, (2), pp. 87-94, 1999.

[10] N. Abhayasinghe and I. Murray, "Human gait phase recognition based on thigh movement computed using IMUs," 2014 IEEE Ninth International Conference on Intelligent Sensors, Sensor Networks and Information Processing (ISSNIP), Singapore, 2014, pp. 1-4. doi: $\quad$ 10.1109/ISSNIP.2014.6827604 URL: http://ieeexplore.ieee.org/stamp/stamp.jsp?tp=\&arnumber=682760 $4 \&$ isnumber $=6827478$

[11] H. J. Luinge and P. H. Veltink, "Measuring orientation of human body segments using miniature gyroscopes and accelerometers," Medical and Biological Engineering and Computing, vol. 43, (2), pp. 273-82, 2005. DOI: http://dx.doi.org.dbgw.lis.curtin.edu.au/10.1007/BF02345966.

[12] K. P. Hopkins, "Low Cost AHRS and GPS/IMU Integration using Kalman Filtering for Autonomous Vehicle Navigation." Order No. 1508359, University of Rhode Island, Ann Arbor, 2012.

[13] S. O. H. Madgwick, A. J. L. Harrison and R. Vaidyanathan, "Estimation of IMU and MARG orientation using a gradient descent algorithm," 2011 IEEE International Conference on Rehabilitation Robotics, Zurich, 2011, pp.

$1-7$. doi:

10.1109/ICORR.2011.5975346 URL: http://ieeexplore.iee.. rg/stamp/stamp.jsp?tp=\&arnumber=597534 6\&isnumber $=5975334$

[14] D. Roetenberg, H. J. Luinge, C. T. M. Baten and P. H. Veltink, "Compensation of magnetic disturbances improves inertial and magnetic sensing of human body segment orientation," in IEEE Transactions on Neural Systems and Rehabilitation Engineering, vol. 13, no. 3, pp. 395-405, Sept. 2005 doi: $\quad 10.1109 /$ TNSRE.2005.847353 URL: http://ieeexplore.iee.org/stamp/stamp.jsp?tp=\&arnumber=150682 $5 \&$ isnumber $=32284$

[15] Wu, Z. Sun, W. Zhang, and Q. Chen. Attitude and gyro bias estimation by the rotation of an inertial measurement unit. http://iopscience.iop.org/article/10.1088/0957-0233/26/12/125102/pdf

[16] V. Bonnet, J. McCamley, C. Mazza and A. Cappozzo, "Trunk orientation estimate during walking using gyroscope sensors," 2012 4th IEEE RAS \& EMBS International Conference on Biomedical Robotics and Biomechatronics (BioRob), Rome, 2012, pp. 367-372. doi: $\quad$ 10.1109/BioRob.2012.6290821 URL: http://ieeexplore.ieee.org/stamp/stamp.jsp?tp=\&arnumber=629082 $1 \&$ isnumber $=6290255$

[17] R. S. McGinnis et al., "Accuracy of Femur Angles Estimated by IMUs During Clinical Procedures Used to Diagnose Femoroacetabular Impingement," in IEEE Transactions on Biomedical Engineering, vol. 62, no. 6, pp. 1503-1513, June 2015. doi: 10.1109/TBME.2015.2392758

[18] Okita, Nori, and H. Sommer. "Angular acceleration of the foot during gait using an IMU." Annual Meeting of American Society of Biomechanics $(A S B) .2013$

[19] T. N. Do, R. Liu, C. Yuen, M. Zhang and U. X. Tan, "Personal Dead Reckoning Using IMU Mounted on Upper Torso and Inverted Pendulum Model," in IEEE Sensors Journal, vol. 16, no. 21, pp. 7600-7608, Nov.1,
2016

doi: 10.1109/JSEN.2016.2601937

[20] P. K. Yoon, S. Zihajehzadeh, B. S. Kang and E. J. Park, "Robust Biomechanical Model-Based 3-D Indoor Localization and Tracking Method Using UWB and IMU," in IEEE Sensors Journal, vol. 17, no. 4, $\begin{array}{llll}\text { pp. 1084-1096, } & \text { Feb.15, } & 15 & \end{array}$ doi: 10.1109/JSEN.2016.2639530

[21] M. Nordin and V. H. Frankel, Basic Biomechanics of the Musculoskeletal System. Lippincott Williams \& Wilkins, 2001, pp. 439.

[22] J. Perry and J. M. Burnfield, Gait Analysis: Normal and Pathological Function 2nd Edition, Thorofare, New Jersey: SLACK Incorporated, 2010

[23] K. Kawano, S. Kobashi, M. Yagi, K. Kondo, S. Yoshiya and Y. Hata, "Analyzing 3D Knee Kinematics Using Accelerometers, Gyroscopes and Magnetometers," 2007 IEEE International Conference on System of Systems Engineering, San Antonio, TX, 2007, pp. 1-6. doi: 10.1109/SYSOSE.2007.4304332

[24] M. Henriksson. (2013, Sept 16). Estimation of Heading using Magnetometers and GPS. [Online]. Available: https://pdfs.semanticscholar.org/98f3/5084669a7d3926ea96e21e9389c1 ce51661c.pdf

[25] H. Nguyen, K. Lebel, S. Bogard, E. Goubault, P. Boissy and C. Duval, "Using Inertial Sensors to Automatically Detect and Segment Activities of Daily Living in People With Parkinson's Disease," in IEEE Transactions on Neural Systems and Rehabilitation Engineering, vol. 26, no. $1, \quad$ pp. 197-204, Jan. 2018. doi: 10.1109/TNSRE.2017.2745418

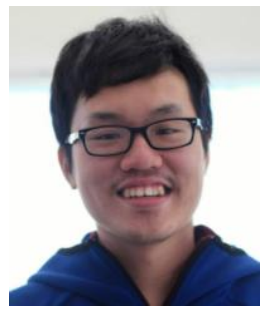

Yi Chiew Han received the B.Eng. (Hons.) in electronic and communication engineering from Curtin University Australia in 2015. He is currently working toward the $\mathrm{PhD}$ degree at Curtin University Malaysia. His research interests include designing wearable sensor devices to understand more of human ambulation.

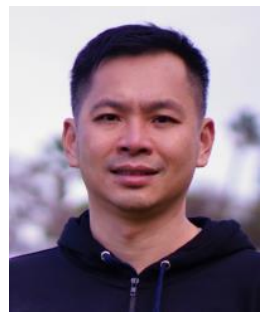

Kiing Ing Wong was born in Sarawak, Malaysia, in 1975. He received the B.Eng. degrees in electrical and electronic engineering from the University of Manchester, U.K., in 1999 and the Ph.D. degree from Southampton University, U.K., in 2004. He is a senior lecturer at Curtin University, Malaysia. His research interests involve designing electronic devices.

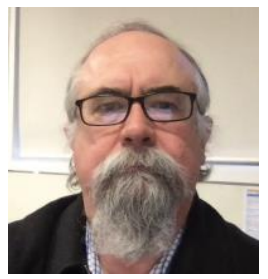

Professor Iain Murray AM received his B.Eng (Hons) in Computer Systems Engineering in 1998 and his $\mathrm{PhD}$ titled "Instructional eLearning technologies for the vision impaired" in 2008, both at Curtin University. He has worked in the field of assistive technology for more than 25 years both as a practitioner and researcher. Currently employed as Engineering Curriculum Lead in the Faculty of Science and Engineering, his research interests include learning environments for people with vision impairment, embedded sensors in health applications and assistive technology. $\mathrm{He}$ founded the "Cisco Academy for the Vision Impaired" in 2002 to deliver ICT training to vision impaired people globally. He has supervised 17 research students' completions and published in excess of 110 peer reviewed articles. He is a Member of the Order of Australia, Fellow of the Australian Computer Society, Curtin Academy Fellow and a senior member of the IEEE. 